\title{
Developing the role of the nurse specialist
}

\section{Expanding the role of the nurse in the Accident and Emergency department}

\author{
Barbara L Neades
}

\begin{abstract}
Summary
In response to the increasing demands upon the Accident and Emergency department and supported by changes within the scope of professional practice of the qualified nurse, Accident and Emergency nurses have expanded their role within the multidisciplinary team. The article reviews the development of this expanded role for the nurse within the Accident and Emergency team and discusses its implications.
\end{abstract}

Keywords: nurse, Accident and Emergency medicine
Despite government initiatives to improve the primary care system within the UK, ${ }^{1}$ Accident and Emergency (A\&E) department attendances continue to rise. The National Audit Office indicated that in 1990/91 11.2 million new attenders were seen in England and 1.2 million in Scotland. ${ }^{2,3}$

Jones $^{4,5}$ highlights how seven out of nine national Patients' Charter standards ${ }^{6}$ have been written specifically for A\&E departments, suggesting the need for the development of new strategies of care for the patient within A\&E. In addition, the Calman report, ${ }^{7}$ government strategies to reduce junior doctors' hours ${ }^{8}$ and the publication of the Scope of professional practice for nursing, midwifery and health visiting, ${ }^{9}$ have arguably changed the face of $A \& E$ services within the UK.

The most controversial of these changes has been the expansion of the role of the nurse within the $A \& E$ department. ${ }^{10} \mathrm{~A}$ debate has ensued as to the appropriateness of the changes in medical and nursing roles in an effort to improve care in A\&E. Some professionals welcome the changes which have taken place within nurse education and practice, which allow the development of the nurse's knowledge and skills. ${ }^{11,12}$ Others, however, lament the changes occurring within nursing, suggesting that there has been an erosion of traditional nursing values such as care, compassion and loyalty. ${ }^{13-15}$ This paper reviews the development of the role of the nurse specialist within the A\&E multidisciplinary team, examining some of the issues raised by these developments.

\section{The nurse specialist in A\&E}

In the light of government guidelines on extended roles, ${ }^{16} \mathrm{~A} \& \mathrm{E}$ nurses have adapted their role to include tasks previously undertaken by medical staff, including suturing wounds, plastering fractures, and recording of electrocardiograms. Jones ${ }^{17}$ found that normal practice for nurses in these departments was impossible to establish, such was the variety of skills being undertaken. The training and legal cover provided by health authorities across the country was similarly varied. Other authors report the routine involvement of experienced $A \& E$ nurses in the triage of $A \& E$ patients, ${ }^{18} \mathrm{X}$-raying of patients with potential fractures, ${ }^{19,20}$ and cannulation of patients. ${ }^{21}$

The practice of these 'extended roles', however, is controlled by protocols established by individual health authorities. Nurses have reportedly felt their practice was curtailed by the need to achieve certificates of competence for each individual extended role. ${ }^{22}$ The introduction of the Scope of professional practice, ${ }^{9}$ however, has recognised the need for nurses to have a broad area of knowledge and skills to meet the demands of society. In this document, nurses are instructed that their practice must be 'sensitive, relevant and responsive to the needs of individual patients'. Utilising the six principles of practice laid down within this document (box 1), together with the Code of Professional Conduct, ${ }^{23} \mathrm{~A} \& \mathrm{E}$ nurses are now free to identify potential areas of expansion to their practice which they consider to be beneficial to patient care. The UK Central Council (UKCC), ${ }^{9}$ however, highlights the requirement for nurses to adhere to a safe standard of care and competence, gaining suitable experience and education prior to expansion of practice.

The National Health Service (NHS) Management Executive ${ }^{24}$ recognised the benefits of utilising the skills of nurses and health visitors to enhance the quality of care. This review of the boundaries of practice was welcomed by $A \& E$ nurses whose attempts to expand their role had often been frustrated by existing legislation. ${ }^{6}$ The support and freedom contained within the $S$ cope of professional practice has allowed $A \& E$ nurses to make a greater contribution to the multidisciplinary team. However, there is still considerable debate about the appropriate role of the nurse in A\&E. 


\section{Triage priority system}

Priority 1: patients who require immediate attention

la: standby, immediate medical intervention on arrival

1b: must be seen by medical staff

within 15 minutes of arrival

Priority 2: must be seen by medical staff within one hour of arrival

2a: need on medical grounds

$2 b$ : need on social grounds

Priority 3: can safely wait up to three hours for medical attention

Priority 4: while these can wait indefinitely for treatment, a maximum of four hours has been established

Box 2

\section{Triage in A\&E}

The advancement of nurse triage is one of the main objectives of the Royal College of Nursing's A\&E Nursing Association, ${ }^{25}$ and has been called one of the most important developments in A\&E departments within the past decade. ${ }^{5}$ Triage of $A \& E$ patients, ie, the assessment of patients on arrival to determine their priority of care, was adapted from military medical practice. ${ }^{26}$ This system of prioritisation (box 2), permits life-saving measures, ${ }^{27}$ first-aid, and/or the initiation of diagnostic measure ${ }^{28}$ to be made appropriately. In addition, it can reduce patient anxiety and frustration by providing prompt assessment and information to patients, relatives and friends..$^{29,30}$ It has also been suggested that the procedure makes the best use of $A \& E$ department resources, by directing the patient to the most appropriate care area. ${ }^{30}$

Immediate assessment on arrival in A\&E now forms the basis of one of the quality standards within the Patients' Charter. The term 'immediate' has been criticised as being impractical and, although clarified as meaning within five minutes of arrival, it remains controversial.

A debate also exists as to how, when, and where triage occurs. Crouch ${ }^{31}$ identifies three methods of triage:

- before registration of the patient

- with registration of the patient

- after registration of the patient.

Blythin ${ }^{27}$ advocates the application of the first method of triage, which requires the triage nurse to be situated in or near the waiting area and undertake a preliminary assessment of the patient. This method is favoured by many practitioners in an attempt to achieve Charter standards. Other practitioners have adopted a combined triage and registration occurring within the waiting area.

The triage nurse has a responsibility to record any assessments made of the patient's needs. ${ }^{23}$ Some authors have estimated that it can take up to 10 minutes to complete a full triage history. ${ }^{32}$ This delay may result in failure to achieve the Patients' Charter standard.

To address this requirement some departments have developed a two-stage system of triage. The first stage entails a brief history taking and superficial assessment of the patient by an experienced nurse to identify any immediate requirement for care. Brief documentation occurs here. Following registration, the patient undergoes an in-depth assessment by another nurse, with more extensive documentation taking place. Two immediate problems can be identified with this triage system. If the first triage nurse meets the Patients' Charter standards but breaches the Code of Conduct by not adequately documenting the patient's problems, the nurse's accountability for practice is surely questionable.

Another problem surrounding the triage of patients within the waiting area relates to the confidentiality of patient information. Even the briefest of assessments entails the triage nurse requesting personal information from the patient. Unless there is a specially allocated area within the waiting area, this confidential information may be overheard by other patients or relatives. This may constitute a breach of confidentiality.

To alleviate these difficulties, other practitioners have adopted a system where the patient is registered first and then proceeds to a specially designed area where a full triage assessment is undertaken and documented. This may result in the department falling short of the Charter standard. Supporters of this method of triage acknowledge this difficulty, but suggest that the benefits in terms of quality outweigh the problems of patients not being assessed 'immediately'.

The benefits of triage in relation to the reduction of patient waiting times in $\mathrm{A} \& \mathrm{E}$ has also been the subject of considerable debate. Some observers ${ }^{33}$ suggest that triage can reduce overall waiting times for $A \& E$ patients. It is also suggested that triage reduces anxiety and dissatisfaction of patients and relatives with lower priorities of care, by allowing them early access to a qualified nurse for assessment. ${ }^{29}$ Other studies, ${ }^{34,35}$ however, challenge the role of triage in reducing waiting times to treatment, suggesting that it may actually increase waiting times. Some also suggest that there is no discernible difference in patient satisfaction between groups of patients who are triaged and those who are not. It is acknowledged, however, that these findings may only reflect local practices.

Geraci and Geraci, ${ }^{36}$ in investigating factors which contributed to delayed triage of patients, supported the theory that inadequate staff resulted in extended triage times. The triage nurse becoming involved in what they described as non-primary triage functions during triage (eg, answering 


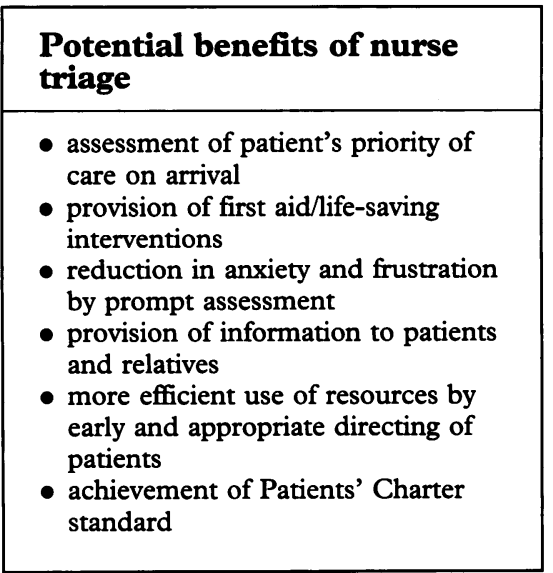

Box 3 telephone requests for advice, interruptions from other healthcare professionals and giving directions and retrieving records for medical and other staff), had a major influence on poor triage time outcomes.

At the heart of this debate lie the fundamental goals and potential benefits of triage (box 3). Triage can also be viewed as an opportunity for the nurse to communicate with the patient and family, providing information as well as health promotion and accident prevention advice, with emphasis on the quality of contact with the patient. Others view triage as a function of the $A \& E$ department's attempts to achieve the Charter standard of assessment of all patients within five minutes of arrival.

The A\&E specialist must therefore consider whether their application of triage is providing the envisaged improvements in the quality of patient care or merely addressing the Charter requirements in terms of time.

\section{Telephone triage}

A further development in $\mathrm{A} \& \mathrm{E}$ nursing has been the establishment of an extended triage system utilising the telephone. ${ }^{37}$ Evans et $a l,{ }^{38}$ note how the public often telephone the A\&E department for advice. Telephone triage involves the nurse providing advice to the public on health-related matters. In addition, decisions regarding appropriate first aid, the need for the patient to seek professional healthcare or ability to self-care, may also be made by the nurse. ${ }^{39}$ Wilkins (unpublished) has highlighted the financial savings to the healthcare system that can be obtained by instigating such a service.

Some practitioners, however, question the accountability related to this practice within A\&E nursing. ${ }^{38,40,41}$ They highlight how some telephone triage systems appeared to operate on a very ad hoc basis, with little reference to structured protocols or documentation. Edwards ${ }^{41}$ explored the diagnostic reasoning utilised by nurses during telephone triage. He found that nurses utilised a systematic approach to making decisions on appropriate patient advice, and rejected the requirement for formal telephone triage protocols. Other authors dispute this, however, suggesting that, when protocols of practice are not utilised, some of the information provided could be inappropriate and even harmful to the patient. ${ }^{38,40,42}$ Given the variety of requests for advice received in the $A \& E$ department, they question the triage nurse's ability to comment adequately and safely on every care aspect without the aid of some form of acceptable protocol.

Dale $e t a l^{40}$ also support the need for protocols and additional education for the nurse, highlighting the stress associated with being inadequately prepared. Education is crucial in dealing with situations requiring crisis intervention skills, for example, in dealing with an attempted suicide. These authors advocated the use of well-developed protocols of practice and have devised a computer data base of information which could be used as part of a telephone triage system.

Telephone triage has the potential to enhance greatly the quality of care provided by the A\&E department. However, it is not the panacea for all ills. Its development can only be achieved by multidisciplinary planning, appropriate resources and provision of adequate numbers of suitably prepared staff.

\section{The Emergency Nurse Practitioner}

Another initiative within $A \& E$ nursing has been the development of Emergency Nurse Practitioner systems throughout the UK. These have been welcomed by the medical profession, ${ }^{43,44}$ the government ${ }^{45}$ and the National Audit Office, ${ }^{20}$ seeing their potential to cut waiting times, improve care and reduce pressure on junior doctors in A\&E (box 4). $\mathrm{Kohn}^{46}$ documented the establishment of the first Emergency Nurse Practitioner service in this country and a number of such schemes have now been established. ${ }^{47,48}$

Each $A \& E$ scheme includes a variety of roles, for example, diagnosis and treatment of minor injuries, referral to other health professionals, and health promotion activities. The Royal College of Nursing ${ }^{49}$ has attempted to define this role (box 5). In some practices, for example, some diagnostic tests (eg, Xrays) may be requested by the Emergency Nurse Practitioner prior to the patient being seen by the doctor..$^{50}$ The health promotion aspects of the role are also highlighted, demonstrating how the Emergency Nurse Practitioner's knowledge and skills can be further utilised to care for vulnerable groups within inner city areas (Crouch, personal communication). The scope of practice of this role, however, varies according to local requirements. In some areas the Emergency Nurse Practitioner is employed solely in this role, allowing development of specialist practice areas. The Emergency Nurse 


\begin{tabular}{|l|}
\hline The Emergency Nurse \\
Practitioner: definition \\
\hline An Emergency Nurse Practitioner in \\
A\&E is a nurse who has a sound \\
nursing practice based on all aspects of \\
A\&E nursing, with formal post-basic \\
education in holistic assessment, \\
physical diagnosis, prescription of \\
treatment and promotion of health. \\
The Emergency Nurse Practitioner is a \\
key member of the healthcare team \\
directly available to members of the \\
public. He/she must be an autonomous \\
practitioner, able to assess, diagnose, \\
treat and discharge patients without \\
reference to a doctor, but within pre- \\
arranged guidelines. He/she must be \\
able to make independent referrals to \\
other healthcare professionals \\
\end{tabular}

Box 5

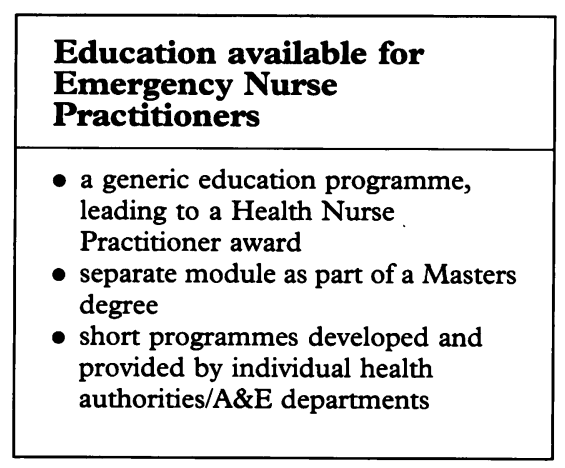

Box 6

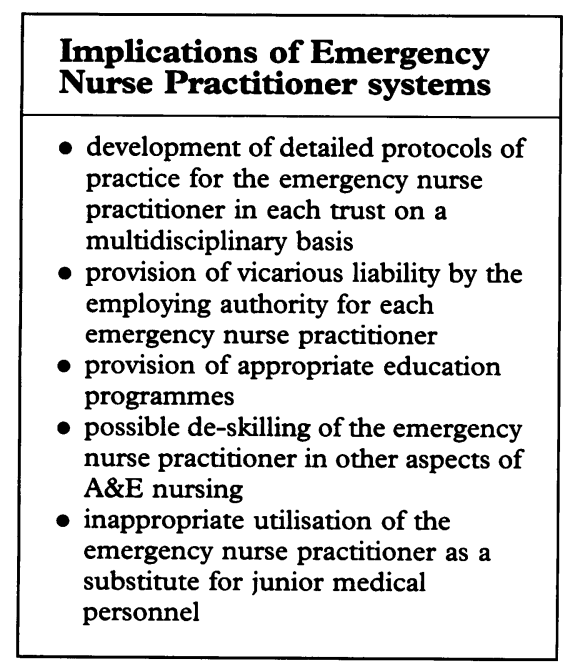

Box 7
Practitioner may undertake such advanced roles as the assessment and treatment of hand injuries, as well as developing a health promotion/accident prevention role within the community (Keltie, personal communication). This may further reduce attendances and waiting times within major $A \& E$ departments.

Critics of this approach suggest that this may mean deskilling of the Emergency Nurse Practitioner in other aspects of A\&E nursing, eg, resuscitation, teaching, and management of the $A \& E$ department. ${ }^{48}$ They favour combining the role with that of the department Sister.

Supported by guidance from the Royal College of Nursing, ${ }^{49}$ some Emergency Nurse Practitioner schemes have now been developed as nurseled minor injuries units. ${ }^{51}$ These units often provide minor injury care following the closure of the major A\&E department. They may also refer patients to onsite physicians, psychiatrists and physiotherapy services, or to the major $A \& E$ department, and may administer medication to the patient according to agreed protocols. There have been problems, however, with the development of this role. Howie $\mathrm{e}^{52}$ highlights the inter-professional difficulties experienced with the Emergency Nurse Practitioner requesting X-rays. Davies ${ }^{10}$ recognised the considerable pressures on the Emergency Nurse Practitioner. She suggests that the role of the Emergency Nurse Practitioner is often seen only in terms of what the nurse can do from a task-orientated perspective and as a doctor substitute to reduce junior doctors' hours.

Some authors have vigorously disputed the suggestion that nurse practitioners should be utilised as junior doctor substitutes. ${ }^{11,53}$ They list direct improvements in the quality of patient care gained from this role, including listening/counselling services, support, and monitoring the health of patients, in addition to the care of their minor injuries.

Castledine $^{54}$ advises that any nurse practitioner carrying out medically orientated procedures should ensure that they adopt an approach 'incorporating a nursing focus of caring, comforting and counselling', lest they become no more than technicians undertaking tasks on behalf of the medical practitioner. Dowling et $a l,{ }^{55}$ also question whether, as a result of the nurse taking on more technical roles, the highly valued nursing characteristics of caring and communication might suffer.

Touche Ross ${ }^{56}$ evaluated the work of nurse practitioners at 20 sites, including A\&E. They reported the improvements in care made by the nurse practitioner, highlighting the provision of safe and effective care. They did suggest, however, that this did not necessarily result in cost reductions.

Dowling et al, ${ }^{55}$ and Wilson ${ }^{57}$ have also raised doubts about the proposed cost savings in expanding the nurse's role to reduce the workload of the junior doctor. They indicated that the limited role developed for some nurse practitioners did not make them cost effective.

To some, the Emergency Nurse Practitioner is the answer to all the problems of the A\&E department. The Emergency Nurse Practitioner cannot only care for the minor injury patient but, in addition, may redirect patients who do not require treatment in the $A \& E$ department. The resolution of the 'inappropriate attender' dilemma is nigh! Cable (unpublished) suggests, however, that these patients' non-requirement for medical care, does not negate the benefits they could accrue from the listening, supportive, and health-promotion skills of the Emergency Nurse Practitioner. He questions the practice of redirecting these patients away from $A \& E$ because they do not require medical intervention.

In response to financial pressures some $A \& E$ managers are now considering substituting the Emergency Nurse Practitioner for the junior doctor in A\&E, with considerable implications for both professions.

In addition to the professional implications of the establishment of an Emergency Nurse Practitioner system, the legal and accountability aspects of the role also require consideration. The need for vicarious liability from the employer necessitates the establishment of detailed protocols of practice for the Emergency Nurse Practitioner whilst in the employ of that particular health authority/trust and is not transferable.

The educational implications of providing this service are also considerable. The Royal College of Nursing ${ }^{49}$ defines the Emergency Nurse Practitioner as a nurse with a sound base in $A \& E$ and appropriate formal post-basic education. The education available for this role varies considerably (box 6). Walsh ${ }^{58}$ suggests the need for a nationally recognised education programme to develop and maintain standards of practice. Developing these courses requires considerable time, finance, and multidisciplinary support.

If the full potential for this role in patient care is to be realised, the Emergency Nurse Practitioner should not be viewed as a replacement for the 
junior doctor, but as a professional with qualities and skills which complement those of the medical staff in A\&E. This is not a quick or cheap solution to the problem of increasing $A \& E$ attendances. ${ }^{53}$ There must be inter-professional discussions to clarify the manpower, educational and professional/political implications of the Emergency Nurse Practioner's role (box 7). In addition, further research to determine the appropriate scope of practice of the Emergency Nurse Practitioner and to establish the position of the Emergency Nurse Practitioner within the multidisciplinary A\&E team must be undertaken if this unique opportunity to enhance $A \& E$ care is not to be missed.

\section{Resuscitation of acutely ill patients}

A\&E nurses now undertake a much more active role within Advanced Life Support and Advanced Trauma Support Resuscitation teams. ${ }^{59,60}$ Lomas and Goodall $^{61}$ suggest that a structured and organised team with predetermined roles and responsibilities for both nursing and medical staff, can have a direct bearing on patient outcomes in resuscitation. Utilising the specialist knowledge and skills gained during the Advanced Life Support Course, ${ }^{62}$ Advanced Trauma Nursing Course, ${ }^{63}$ or the Trauma Nursing Core Course, ${ }^{64}$ experienced $A \& E$ nurses are now making a greater contribution to the care of patients with life-threatening conditions. With these courses, recognised by the Royal College of Surgeons and the Royal College of Nursing, the nurse not only gains theoretical knowledge of the assessment and management of the patient with a cardiac arrest or multiple trauma, but also develops the practical skills needed in resuscitation of these patients.

It is acknowledged that, in the UK, nurses are not often required to undertake advanced resuscitation procedures alone, such as the insertion of chest drains or peritoneal lavage. However, nurses have been required to intubate and cannulate patients in an emergency situation. ${ }^{61}$ The experienced $A \& E$ nurse is also often required to advise, support and sometimes direct junior medical colleagues in an emergency situation, until more senior medical staff can attend. The experienced $A \& E$ nurse must therefore have the appropriate knowledge and skills to support the junior doctor in the application of the Advanced Life Support or Trauma protocols.

Together, these courses provide the nurse with a sound education so that they may undertake a full role within the cardiac or trauma resuscitation team. Indeed, a number of senior nurses are now certified instructors in Advanced Life and Trauma Support. Utilising the knowledge and skills of the experienced nurse in this manner could be viewed as an effective use of department resources, allowing considerable improvements in the care of the critically ill cardiac or trauma patient to be achieved. ${ }^{59,65}$ Gautam and Heyworth, ${ }^{66}$ however, claim that these courses are often inaccessible to nursing staff due to their limited availability and the lack of funding. Hadfield-Law ${ }^{65}$ suggests that, unlike the medical staff who attend these courses, many nurses have difficulties in obtaining funding and study leave. Conaghan ${ }^{67}$ suggests that these courses are only the first step to improving care, and highlights how quickly skills deteriorate after acquisition. She suggests that, without follow-up, practice and support, these courses are a waste of money. Practice may be gained via access to mannequins or, alternatively, by the utilisation of these skills on a regular basis, within a cardiac arrest or specialist trauma team.

Despite the availability of these educational courses and the publication of the Scope of professional practice, few senior A\&E nurses who hold the appropriate qualification are utilised fully as part of such teams. Hospital protocols still often insist that medically qualified personnel should co-ordinate resuscitation attempts, even if the medically qualified person is a junior doctor with limited experience in the field.

There is, of course, a legal requirement for a doctor to take responsibility for certain procedures, such as the prescription of drugs. If, however, the nurse is required to administer a drug within an agreed protocol, the employing authority can draw up an agreement with the nurse where vicarious liability will be extended. This practice is limited, however. This situation raises some questions as to the perception of the experienced nurse by the other members of the multidisciplinary team and the appropriate utilisation of this highly educated and expensive $A \& E$ resource.

If improvements in the resuscitation of critically ill patients are to continue, the development of multidisciplinary teams possessing advanced skills is essential. The experienced, trained, A\&E nurse is an integral part of that team. Perhaps as a result of previous legislation and education we are not making the best use of this resource. Clearly, this area merits attention, including a reconsideration of professional and personal perceptions. 
1 Department of Health. Working for patients. London; HMSO, 1989

2 National Audit Office (NAO). NHS Accident and Emergency Departments in England. London; NAO, 1992.

3 National Audit Office (NAO). NHS Accident and Emergency Departments in Scotland. London: NAO, 1992

4 Jones G. The Patients' Charter in the Accident and Emergency Department. Accid Emerg Nurs and Emergency

5 Jones G. Accident and Emergency and the Emergency Nurse Practitioner. In: Hunt G Emergency Nurse Practitioner. In: Hunt G,
Wainwright P, eds, Expanding the role of the Wainwright P, eds, Expanding
nurse. London: Blackwell, 1994.

6 Department of Health. The Patients' Charter London: HMSO, 1991

7 The Working Group on Specialist Medical Training. (K Calman Chairman). Hospital doctors: training for the future. London: $\mathrm{DOH}, 1993$.

8 National Health Service Management Executive. Funior Doctors: the new deal. London: $\mathrm{DOH}$ 1991.

9 United Kingdom Central Council for Nursing, Midwifery and Health Visiting (UKCC). The scope of professional practice. London: UKCC, scope
1992.

10 Davies J. Expanding horizons. Nurs Times 1992; 88 (47): $37-9$.

11 Stillwell $B$. The rise of the nurse practitioner. Community Outlook 1992; 2 (11): 16-9.

12 Savage J. What is happening to nursing. $B M$ 1995; 311: $274-5$

13 Hay R. A nurse's place is at the bedside. Nur Stand 1994; 8 (27): 42-3.

14 Short TA. A dual perspective. BMF 1995; 311 303-4.

15 Bradshaw A. Nursing and medicine: co-operation or conflict? BMF 1995; 311: 304-5.

16 Department of Health and Social Security (DHSS). The extending role of the clinical nurse. Legal implications and training requirements. London: DHSS, 1977 (HC (77) 22).

17 Jones G. Behind the times. Nurs Times 1986; 82 (42): $30-3$.

18 Mallet J, Woolwich C. Triage in Accident and Emergency Departments. $\mathcal{J}$ Adv Nurs 1990; 15 $1443-51$

19 MacLeod AJ, Freeland P. Should nurses be allowed to request $\mathrm{X}$-rays in an Accident and Emergency Department? Arch Emerg Med 1992 9: $19-22$.

20 Davies J. X-Ray vision of shorter queues. Nurs Times 1994; 90 (21): $52-4$

21 Denner S. Extending professional practice: benefits and pitfalls. Nurs Times 1995; 91 (4): 27-9.

22 Rea K. The UKCC code deciphered. Nurs Stand 1992; 6 (51): $51-4$.

23 United Kingdom Central Council for Nursing, Midwifery and Health Visiting. Code of profesMidwifery and Health Visiting. Code
sional conduct. London: UKCC, 1992.
24 National Health Service Management Executive. Hours of work of doctors in training: making the best use of the skills of nurses and midwives London: DOH 1992.

25 Jones G. A\&E nursing today and the future. Nurs Stand 1990; 4(27): 51-2.

26 Crouch $R$. Inappropriate attender in A\&E. Emerg Nurse 1992; 6 (27): 7-9.

27 Blythin P. Triage: a nursing care system. In: Wright $\mathrm{B}$, ed. Management and practice in emergency nursing London: Chapman Hall 1988.

28 Williams DG. Sorting out triage. Nurs Times 1992; 88 (30): $34-6$.

29 Buckles E. Evaluation of patient satisfaction in A\&E. Nurs Stand 1990; 4 (19): 33-5.

30 Bailey A, Hallam $K$, Hurst $K$. Triage on trial Nurs Times 1987; 83 (44): $65-6$

31 Crouch R. Triage past, present and future. Emerg Nurse 1994; 1 (2): 4-6.

32 Estrada EG. Triage systems. Nurs Clin North Am $1981 ; 1$ (16): $13-24$

33 Wong TW, Tseng G, Lee W. Report of an audit of nurse triage in an Accident and Emergency department. Accid Emerg Med 1994; 11: 91-5.

34 George S, Read S, Westlake L, Williams B, Fraser-Moodie A, Pritty P. Evaluation of nurse triage in a British Accident and Emergency triage in a British Accident and Em

35 Shields JE. Making triage work: the experience of an urban emergency department. $\mathcal{F}$ Emerg Nurs 1976; 2: 37-41.

36 Geraci EB, Geraci TA. An observational study of the emergency triage nursing role in managed care facility. $\mathcal{F}$ Emerg Nurs 1994; 20 (3): $189-94$

37 Buckles E, Carew-McColl M. Telephone triage. Nurs Times 1991; 87 (6): 26-8.

38 Evans RJ, McCabe M, Richmond P. Telephone advice in Accident and Emergency: a survey of current practice. Arch Emerg Med 1993; 10: 216-9.

39 Glasper A, McGrath K. Telephone triage extending practice. Nurs Stand 1993; 7 (15): 34-6.

40 Dale J, Williams S, Crouch R. Development of telephone advice in A\&E: establishing the views of staff. Nurs Stand 1995; 9 (21): 28-31.

41 Edwards B. Telephone triage: how experienced nurses reach decisions. $\mathcal{f}$ Adv Nurs 1994; 19: 717-24.

42 Glasper A. Telephone triage: a step forward for nursing practice? Brf Nurs 1992; 2 (2): $107-8$.

43 Yates DW. Nurse practitioners for A\&E. $B r f$ Accid Emerg Med 1987; 3: 10-1.

44 James MR, Pyrogos N. Nurse practitioners in the Accident and Emergency department. Arch Emerg Med 1989; 6: 241-6.

45 Warden J. Rise of the nurse practitioner. BMF 1988; 296: 1478 .

$46 \mathrm{Kohn} \mathrm{P.} \mathrm{Implementing} \mathrm{a} \mathrm{nurse} \mathrm{practitioner}$ scheme. Emerg Nurse 1987; 1 (4): 2 .
47 Potter T. A real way forward in A\&E. Prof Nurse 1990; 5: 586-8

48 Burgess $\mathrm{K}$. A dynamic role that improves the service. Prof Nurse 1992; 7: 301 - 3 .

49 Royal College of Nursing (RCN). Guidance for emergency nurse practitioners. London: RCN, A\&E Association, 1993.

50 Woolich C. A wider frame of reference. Nurs Times 1992; 88 (46): 34-6.

51 Beales J, Baker B. Minor injuries unit: expanding the scope of Accident and Emergency ing the scope of Accident and Emergen

52 Howie P. Development of the nurse practitioner. Nurs Stand 1992; 6 (27): 10-1.

53 Walsh M. Accident and emergency nursing: a new approach, 2nd edn. Oxford: Heinemann, 1990.

54 Castledine G. Nurse practitioner title: ambig uous and misleading. Br $\mathcal{F}$ Nurs 1993; 2 (14): $734-5$.

55 Dowling S, Barrett S, West R. With nurse practitioners who needs house officers? $B M \mathcal{F}$ 1995; 311: 309-11.

56 Touche Ross \& Co. Evaluation of nurse practitioner pilot projects: summary report. London: tioner pilot projects: summ
Touche Ross \& Co, 1994.

57 Wilson S. Report queries savings with doctor nurse shift. $B M \mp$ 1995; 311: 280 .

58 Walsh $M$. NPs: why are they so successful? Emerg Nurse 1995; 3 (2): 4-5.

59 Belson L. ACLS: cardiac care for the nineties. Emerg Nurse 1993; 1 (1): 9-12.

60 Paynter M. Trauma support: revolution in care. Emerg Nurse 1993; 1 (2): 7-9.

61 Lomas GA, Goodall O. Trauma teams vs nontrauma teams. Accid Emerg Nurs 1994; 2: $205-$ 10.

62 Advanced Life Support Working Party. European Resuscitation Council guidelines for advanced life support. BMF 1993; 306: 1589-93.

63 American College of Surgeons (ACS). Advanced Trauma Life Support Manual. Chicago: ACS, Trauma

64 Emergency Nurses Association (ENA). Trauma Nursing Core Course. Chicago: ENA, 1993.

65 Hadfield-Law L. The development of trauma education for A\&E nurses in the UK. In: Sbaih $\mathrm{L}$, ed. Issues in accident and emergency nursing. London: Chapman Hall, 1994.

66 Gautam V, Heyworth J. The value of the abbreviated ATLS course for accident and emergency nurses. Accid Emerg Nurs 1994; 2 : $100-2$.

67 Conaghan P. ALS skill retention: a cause for concern? Emerg Nurse 1995; 3 (1): 23-5. 